\title{
Morphology and luminescence of photo-electrochemically synthesized porous silicon: Influence of varying current density
}

\author{
Asad Thahe a,b, Hazri Bakhtiar b, ${ }^{\star}$, Noriah Bidin ${ }^{b}$, Zainuriah Hassan ${ }^{c}$, Zainal Abidin Talib d, Uday \\ Basheer ${ }^{\text {, }}$, Dauda Abubakar ${ }^{\circ}$, Muhammad Aizi Mat Salim ${ }^{\mathrm{b}}$, Motahher Abdallah Qaeed ${ }^{\mathrm{f}}$, Hasan \\ Alqaraghuli $g$
}

a Department of Physics, Faculty of Science, Universiti Teknologi Malaysia, 81310 Johor Bahru, Malaysia

b Laser Center, Institute Ibnu Sina, Universiti Teknologi Malaysia, Skudai 81310 Johor, Malaysia

c Institute of Nano Optoelectronics Research and Technology (INOR), Universiti Sains Malaysia, 11800 USM Penang, Malaysia

d Department of Physics, Faculty of Science, Universiti Putra Malaysia (UPM), 43400 Serdang, Selangor, Malaysia

e UTM Centre for Low Carbon Transport, Institute for Vehicle and Engineering, Universiti Teknologi Malaysia, 81310 Johor Bahru, Malaysia Faculty of Education, Department of Physics, Hodeidah University, Al Hodeidah, Yemen

$g$ Faculty of Electrical Engineering, Mechatronics and Automatic Control Department, Universiti Teknologi Malaysia, 81310 Johor Bahru, Malaysia

* Corresponding author: hazri@utm.my

Article history

Received 26 January 2017

Accepted 6 December 2017

\begin{abstract}
Achieving high quality porous silicon (PSi) materials with desired porosity remains challenging. Three good qualities of PSi samples are prepared by Photo electro-chemically etching a piece of $n$-type $\mathrm{S}$ inside the solution of $20 \mathrm{M} \mathrm{HF}, 10 \mathrm{M} \mathrm{C}_{2} \mathrm{H}_{5} \mathrm{OH}$ and $10 \mathrm{M} \mathrm{H}_{2} \mathrm{O}_{2}$ at fixed etching time duration (30 min) and varying current density $\left(15 \mathrm{~mA} / \mathrm{cm}^{2}, 30 \mathrm{~mA} / \mathrm{cm}^{2}\right.$ and $\left.45 \mathrm{~mA} / \mathrm{cm}^{2}\right)$. As-prepared sample morphologies are characterized via scanning electron microscopy (FESEM) and atomic force microscopy (AFM). The gravimetric method is used to estimate the thickness and porosity of the prepared samples. Current density (etching time) dependent morphologies, electronic bandgap and room temperature photoluminescence $(\mathrm{PL})$ properties of such PSi nanostructures are evaluated. These PSi structures revealed enhanced rectifying characteristics with increasing current density.
\end{abstract}

Keywords: Porous Si, morphology, photo-electro-chemical etching, photoluminescence, band gap

\section{INTRODUCTION}

During last two decades the research on porous silicon (PSi) received renewed interests due to the feasibility of opto-electronic applications (Batool et al., 2016; Bisi et al., 2000). Such PSi structures possess distinct room temperature photoluminescence in the visible regions of the electromagnetic spectrum (Pap et al., 2006). They are widely applied in the field of micro-electronics, opto-electronics (Nahor et al., 2011), chemicals manufacturing (Canham, 1990), biological sensors (Lazarouk et al., 1996) and in biomedical devices (Steiner and Lang, 1995) though the integrated circuits industry is dominated by crystalline silicon (CSi). The anodization of singlecrystal Si has resulted in an extensive array of mechanical sensors because of its intrinsic properties such as direct energy band gap (Batool et al., 2016). Although microporous Si presents a very high specific surface (Hussein et al., 2016) but PSi has distinct optical properties different from the bulk one and maintains the crystallinity of the substrates.

Despite much development there is a constant demand for fabricating high quality PSi with desired porosity. Driven by this need, we prepared PSi using photo-electrochemical etching with varying direct current (DC) densities at constant etching time. This technique offers the oppertunity of synthesizing photoluminescent materials with uniform pores and selective wavelength emission. The morphology and PL spectral properties of such synthesized PSi are determined as a function of current density. It is demonstrated that the anodization current density play a significant role in controlling the etching ratio and morphology of the PSi specimen. Present systematic method may constutute a basis for the production of high quality PSi .

\section{MATERIALS AND METHODS}

A slightly doped n-type Si (111) wafer was used to prepared the PSi using photo- electrochemical etching method. Prior to the etching process, the substrates were washed in an ultrasonic bath containing acetone solution, subsequently rinsed with deionized water, and then dried under pure nitrogen gaseous atmosphere. The entire etching procedure was conducted in a Teflon cell. The Si wafer was used as an anode while a Pt mesh was employed as the counter electrode which was linked to the external DC power supply. The electrolyte was comprised of a mixture of $48 \% \mathrm{HF}, 99.90 \%$ ethanol and $30 \% \mathrm{H}_{2} \mathrm{O}_{2}$ with the mole ratio of 2:1:1. Ethanol was added to the aqueous HF solution to increase the wettability of the $\mathrm{Si}$ surface and to eliminate the $\mathrm{H}_{2}$ bubbles generated on the $\mathrm{Si}$ interface. This in turn, enhanced the homogeneity of the PSi layer. The experiment was conducted at a steady etching duration of $30 \mathrm{~min}$ for anodization current densities of 15,30 and $45 \mathrm{~mA} / \mathrm{cm}^{2}$. The front surface of Si was irradiated using a halogen lamp ( $300 \mathrm{~W}$ ) source to increase the concentration of holes on the surface. These holes on the Si surface facilitated the dissolution reactions and thus enhanced the etching procedure. Finally, the porous PSi samples that were immersed in ethanol are dried under nitrogen 
flow.

The PL spectra of Psi samples were recorded using Jobin Yvon HR 800 (UV, Edison, NJ, USA) with an $\mathrm{He}-\mathrm{Cd}$ laser $(325 \mathrm{~nm}, 20 \mathrm{~mW}$ ) as excitaton source. The morphological analyses were performed using field emission scanning electron microscopy (FESEM, Leo Supra 50VP, Carl Zeiss, Germany) and atomic force microscopy (AFM). The energy band gap of the samples was obtained from photoluminescence data.

\section{RESULTS AND DISCUSSION}

\section{FESEM and AFM analysis}

Fig. 1(A-C) show the top views of FESEM images for the PSi samples anodized at $15 \mathrm{~mA} / \mathrm{cm}^{2}, 30 \mathrm{~mA} / \mathrm{cm}^{2}$ and $45 \mathrm{~mA} / \mathrm{cm}^{2}$. An enhancement in the porosity with increasing current density is clearly observed. Moreover, the morphology and the thickness of PSi structures were evolved depending on the anodization current density changes. This confirms that the current density has significant impact on the shape and size of the pores. The formation of regular columns of Si nanocrystal with pores (hole) is seen. At $15 \mathrm{~mA} / \mathrm{cm}^{2}$ of current density, the porous Si exhibits relatively few pores (Fig. 1(A)). The sample grown at higher anodization current density of $30 \mathrm{~mA} / \mathrm{cm}^{2}$ (Fig. 1(B)) displayed higher porosity with columnar structure, which is uniform across the entire surface. However, at anodization current density of $45 \mathrm{~mA} / \mathrm{cm}^{2}$, the pore spaces between the columns become relatively smaller and filled with etched $\mathrm{Si}$ but with increased pore volume as shown in Fig. 1(C).

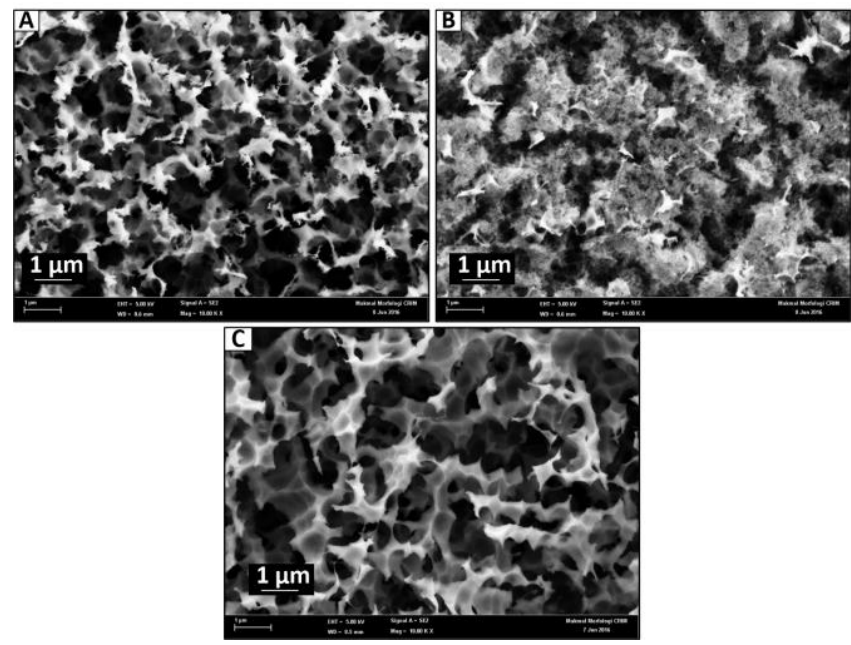

Fig. 1 Top view of FESEM images of synthesized PSi obtained using the current density of: (A) $15 \mathrm{~mA} / \mathrm{cm}^{2}$, (B) $30 \mathrm{~mA} / \mathrm{cm}^{2}$, and (C) $45 \mathrm{~mA} / \mathrm{cm}^{2}$.
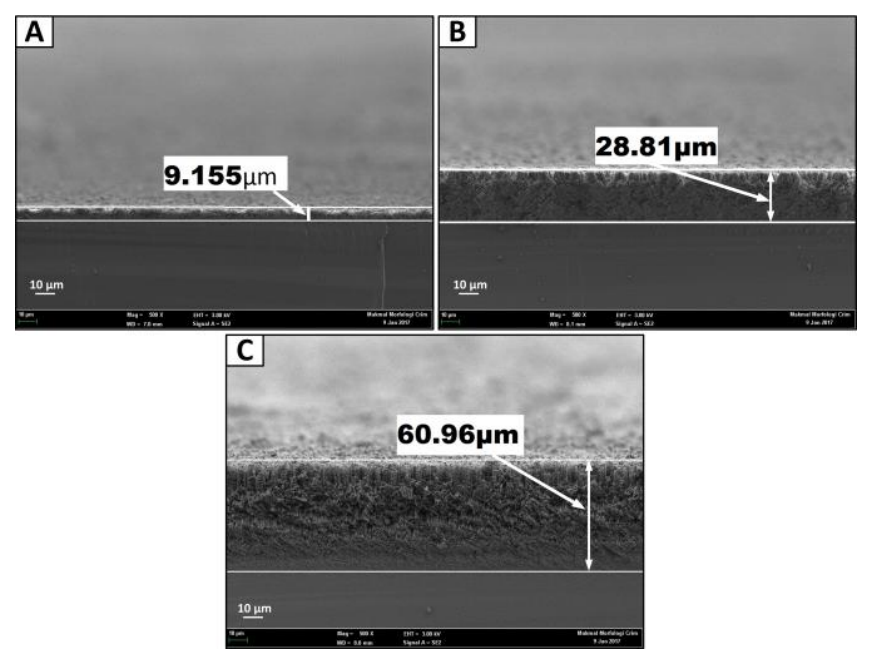

Fig. 2 Cross-sectional view of FESEM images of the PSi obtained at different anodization current density of (A) $15 \mathrm{~mA} / \mathrm{cm}^{2}$, (B) $30 \mathrm{~mA} / \mathrm{cm}^{2}$, and (C) $45 \mathrm{~mA} / \mathrm{cm}^{2}$.
Fig. 2(A-C) illustrate the cross-sectional views of the as-prepared samples. It shows the formation of nonparallel and partially cracked $\mathrm{Si}$ walls with narrow and asymmetrical holes between them. The sample synthesized at anodization current density of $15 \mathrm{~mA} / \mathrm{cm}^{2}$ (Fig. 2(A)) displayed higher degree of nonparallel and partially cracked Si walls of length $9.155 \mu \mathrm{m}$. The sample grown at $30 \mathrm{~mA} / \mathrm{cm}^{2}$ of anodization current density showed substantially wider pores, which are irregular across the entire surface (Fig. 2(B)) with increased thicknesses up to $28.81 \mu \mathrm{m}$. At $45 \mathrm{~mA} / \mathrm{cm}^{2}$ of current density (Fig. 2(C)) these Si walls are differentiated from the sharp pin-shaped holes of lengths of up to $60.96 \mu \mathrm{m}$. Furthermore, an increase in the anodization current density led to a reduction of the column size of the Si NCs.

Fig. 3 depicts the AFM micrographs of all three samples. Sample grown at current density of $15 \mathrm{~mA} / \mathrm{cm}^{2}$ revealed a root mean square (rms) surface roughness of $0.20 \mu \mathrm{m}$ which is increased to $0.23 \mu \mathrm{m}$ and then to $0.57 \mu \mathrm{m}$ for $30 \mathrm{~mA} / \mathrm{cm}^{2}$ and $45 \mathrm{~mA} / \mathrm{cm}^{2}$, respectively. Furthermore, sample synthesized at $45 \mathrm{~mA} / \mathrm{cm}^{2}$ was found to be highly porous having varying shapes such as gouges, tunnels and crevasses.
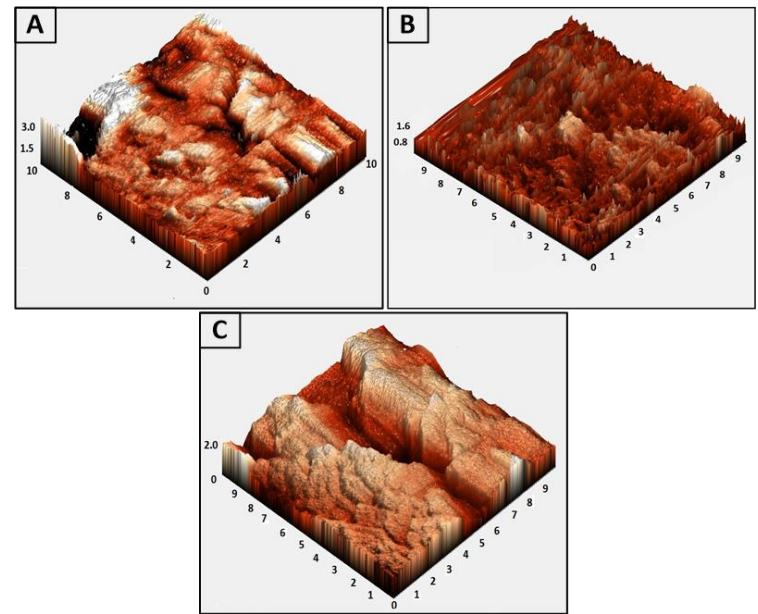

Fig. 3 Cross-sectional view of AFM images of the grown PSi obtained at different anodization current density of: (A) $15 \mathrm{~mA} / \mathrm{cm}^{2}$, (B) $30 \mathrm{~mA} / \mathrm{cm}^{2}$ and (C) $45 \mathrm{~mA} / \mathrm{cm}^{2}$.

\section{Photoluminescence spectra}

Fig. 4 depicts the room temperature PL spectra of various samples. The energy band gap $(\mathrm{Eg})$ values were estimated to be $1.65,1.79$ and $2.06 \mathrm{eV}$ for current densities of $15 \mathrm{~mA} / \mathrm{cm}^{2}, 30 \mathrm{~mA} / \mathrm{cm}^{2}$, and 45 $\mathrm{mA} / \mathrm{cm}^{2}$, respectively. The observed blue shift in the PL peak with increasing anodization current density was attributed to quantum confinement effect (Hirschman et al. 1996), which led to an increase in the energy band gap. The FWHM values for different peaks with increasing current density displayed slight narrowing. The increase in PL intensity with the increase of anodization current density was attributed to the enhanced recombination probability of the carriers that are strongly confined within the nanostructure, where the nature of the band gap is transformed from indirect one to quasi-direct one (Ramizy et al., 2011). The PL peak shift, narrowing of FWHM, and an increase in the peak intensity at $45 \mathrm{~mA} / \mathrm{cm}^{2}$ are consistent with previous findings (Behzad et al., 2012; Abd et al., 2013). The observed S band luminescence is reported to occur within $400-800 \mathrm{~nm}$ (Behzad et al., 2012).

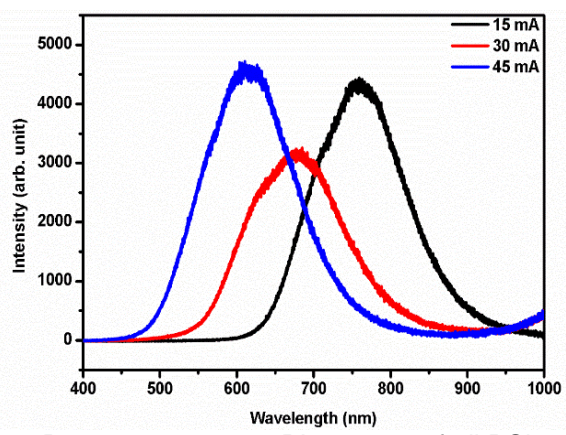

Fig. 4: Room temperature PL spectra of all PSi samples. 


\section{Discussion}

It is asserted that the observed increase in the samples porosity and an enhancement in the PL intensity accompanied by the blue shift with increasing anodization current are strongly correlated. Thus, the variation in the anodization current and etching time duration have strong affect on the light emitting properties of synthesized PSi (Behzad et al., 2012; Abd et al., 2013; Al-Jumail et al., 2016). The increasing porosity of the sample that led to an widening in the energy band gap at higher current density is in good agreement with other reported values (Rajabi and Dariani 2009).

The etched surfaces for samples grown at current density of $30 \mathrm{~mA} / \mathrm{cm}^{2}$, and $45 \mathrm{~mA} / \mathrm{cm}^{2}$ (Fig. B and C in FESEM and AFM images) are irregular and characterized by heterogeneous pores. The pits are obviously formed by an anisotropic etching process, given that the electrolyte disrupts the formation of a passivation layer on the flat etching area faster than the perpendicular sidewalls (Lee et al., 1998). Upon further etching and increased anodization from 15 $\mathrm{mA} / \mathrm{cm}^{2}$ to $45 \mathrm{~mA} / \mathrm{cm}^{2}$, the initial vertical pores are rapidly extended and broadened into gouges and tunnels without forming holes. Furthermore, with increasing anodization the developed pores are progressively interconnected by forming initial undetectable edges of the remaining non-etched layer to appear distinct.

Variation in the anodization current density also influenced the PL intensities, and energy band gap. This enhancement is ascribed to the mounting surface roughness of the PSi nanostructures (Ramizy et al., 2011). Moreover, the observed decreasing and then increasing trend of PL intensity with increasing in the current density is attributed to a large number of dangling bonds in the Psi morphology. This large number of dangling bonds in the PSi exists due to their exposure to the atmosphere which in turn acts as major channel for non-radiative recombination (Abd Rahim et al., 2012; Behzad et al., 2012). Likewise, size distribution is also an important aspect where broader size distribution can cause a drop in the PL intensity (Young et al., 2006; Abd et al., 2013).

\section{CONCLUSION}

The effects of anodization current densities on the morphology and PL spectra of as synthesized PSi were examined. It was demonstrated that by varying the anodization current density it is possible to control the porosity and the visible light emitting properties of such PSi. Good quality PSi samples were achieved. PL spectra revealed prominent peaks together with large blue shift which supported the presence of strong nanoporous structures. Strong surface roughness is established to be responsible for the reduction of the Si crystallite size at higher anodization current density. Based on the results it is affirmed that the efficiency of PSi based photo-detectors can be improved by controlling etching current density assisted porosity.

\section{ACKNOWLEDGEMENT}

The authors would like to express their thanks to the Malaysian government through FRGS vot 4F815 for the finacial support in this project.

\section{REFERENCES}

Abd, H. R. Al-Douri, Y. Ahmed, N. M. and Hashim, U. (2013). Alternativecurrent electrochemical etching of uniformporous silicon for photodetector applications. International Journal of Electrochemical Science, 8(9), pp. 11461-11473.

Abd Rahim, A. F. Hashim, M. R. Rusop, M. Ali, N. K. and Yusuf, R. (2012). Room temperature $\mathrm{Ge}$ and $\mathrm{ZnO}$ embedded inside porous silicon using conventional methods for photonic application. Superlattices and Microstructures, 52(5), pp. 941-948.

Abud, S. H. Hassan, Z. and Yam, F. K. (2014). Fabrication and characterization of metal-semiconductor-metal photodetector based on porous InGaN. Materials Chemistry and Physics, 144(1-2), pp. 86-91.

Batool E. B. Al-Jumaili, Zainal A. Talib, Josephine L. Y., Suriati B. Paiman, Naser M. Ahmed, Abdulmajeed H. J. Al-Jumaily (2016). The correlation of blue shift of photoluminescence and morphology of silicon nanoporous. AIP Conference Proceedings, 1733(1), Article ID 020019.

Behzad, K. Yunus, W. M. M. Talib, Z. A. Zakaria, A. and Bahrami, A. (2012). Effect of preparation parameters on physical, thermal and optical properties of n-type porous silicon. International Journal of Electrochemical Science, 7(9), pp. 8266-8275.

Bisi, O., Ossicini, S., Pavesi, L. (2000). Porous silicon: a quantum sponge structure for silicon based optoelectronics. Surfeace. Science. Reports, 38(13), pp. 1-126.
Canham, L. T. (1990) Silicon quantum wire array fabrication by electrochemical and chemical dissolution of wafers. Applied Physics Letters, 57(10), pp. 10461048 .

Hirschman, K. D., Tsybeskov, L., Duttagupta, S. P., Fauchet, P. (1996). Siliconbased visible light-emitting devices integrated into microelectronic circuits. Nature, 384(6607), pp. 338-341.

Hussein, M. J, Mat Yunus. W. M, Kamari, H, M, Zakaria, A, Oleiw, H. F. (2016). Effect of current density and etching time on photoluminescence and energy band gap of p-type porous Si. Optical and Quantim Electronics, 48, pp. 194.

Lazarouk, S., Jaguiro, P., Katsouba, S., Masini, G., La Monica, S., Maiello, G. and Ferrari, A. (1996) Stable electroluminescence from reverse biased $n$-type porous silicon-aluminum Schottky junction device. Applied Physics Letters, 68(2), pp. 2108-2110.

Lee, M. K., Tseng, Y. C. and Chu, C. H. (1998). A high-gain porous silicon metal-semiconductor-metal photodetector through rapid thermal oxidation and rapid thermal annealing," Applied Physics A: Materials Science and Processing, 67(5), pp. 541-543.

Nahor, A., Berger, O., Bardavid, Y., Toker, G., Tamar, Y., Reiss, L., Sa'ar, A. (2011). Hybrid structures of porous silicon and conjugated polymers for photovoltaic applications. Physica Status Solidi (c), 8(6), pp. 1908-1912.

Nur, H., Hayati, F., Hamdan, H. (2007). On the location of different titanium sites in Ti-OMS-2 and their catalytic role in oxidation of styrene. Catalysis Communications, 8, pp. 2007-2011.

Nur, H., Guan, L. C., Endud, S., Hamdan, H. (2004). Quantitative measurement of a mixture of mesophases cubic MCM-48 and hexagonal MCM-41 by ${ }^{13} \mathrm{C}$ CP/MAS NMR. Materials Letters, 58(12-13), pp. 1971-1974.

Pap, A. E. Kordás, K. Vähäkangas, J. Uusimäki, A. Leppävuori, S. Pilon, L. and Szatmári, S. (2006). Optical properties of porous silicon. Part III: Comparison of experimental and theoretical results. Optical Materials, 28(5), pp. 506-513.

Ramizy, A. Hassan, Z, and Omar. K, (2011). Porous silicon nanowires fabricated by electrochemical and laser-induced etching. Journal of Materials Science: Materials in Electronics, 22(7), pp. 717-723

Steiner, P. and Lang, W. (1995). Micromachining applications of porous silicon. Thin Solid Films, 255, pp. 52-58.

Young, S. J. Ji, L. W. Chuang, R. W. Chang, S. J. and Du, X. L. (2006). Characterization of $\mathrm{ZnO}$ metal-semiconductor-metal ultraviolet photodiodes with palladium contact electrodes. Semiconductor Science and Technology, 21(10), pp. 1507-1511. 